Case Report

\title{
A Case of Ignatzschineria indica Bacteremia following Maggot Colonization
}

\author{
Hugh Muse, ${ }^{1}$ Rachel L. Jenkins, ${ }^{2}$ Meredith B. Oliver, ${ }^{2}$ Soomin Kim, ${ }^{3}$ \\ Richard L. Grantier III, ${ }^{4}$ Bharat K. Malhotra, ${ }^{5}$ Jason J. Parham, ${ }^{6}$ and Kayla R. Stover ${ }^{2}$ \\ ${ }^{1}$ University of Mississippi School of Medicine-Internal Medicine, Jackson, MS 39216, USA \\ ${ }^{2}$ University of Mississippi School of Pharmacy, Jackson, MS 39216, USA \\ ${ }^{3}$ St. Dominic-Jackson Memorial Hospital, Jackson, MS 39216, USA \\ ${ }^{4}$ University of Mississippi School of Medicine-Emergency Medicine, Jackson, MS 39216, USA \\ ${ }^{5}$ Department of Medicine-Hospital Medicine, University of Mississippi Medical Center, Jackson, MS 39216, USA \\ ${ }^{6}$ Department of Medicine-Infectious Diseases, Antimicrobial Stewardship Program, University of Mississippi Medical Center, \\ Jackson, MS 39216, USA
}

Correspondence should be addressed to Kayla R. Stover; kstover@umc.edu

Received 7 September 2017; Accepted 16 October 2017; Published 2 November 2017

Academic Editor: Gernot Walder

Copyright (c) 2017 Hugh Muse et al. This is an open access article distributed under the Creative Commons Attribution License, which permits unrestricted use, distribution, and reproduction in any medium, provided the original work is properly cited.

Ignatzschineria indica is a Gram-negative bacterium that is commonly associated with the larvae of flesh flies. I. indica is difficult to isolate in routine laboratory procedures but has been associated with neglected wounds infested with maggots, fever, elevated white blood count and C-reactive protein, and polymicrobial culture results. Other specific hematological/immunological changes are not known. We present a case of I. indica bacteremia and polymicrobial osteomyelitis resulting from infected decubitus ulcers. The patient improved after treatment with cefepime followed by levofloxacin.

\section{Introduction}

Ignatzschineria indica is a Gram-negative aerobic nonmotile, non-spore-forming rod bacterium that belongs to the family Xanthomonadaceae and the class Gammaproteobacteria [1]. The Ignatzschineria genus is composed of three species (I. indica, I. larvae, and I. ureiclastica) which are common isolates from the larvae of the parasitic flesh fly, Wohlfahrtia magnifica [2]. The genus Ignatzschineria was renamed from Schineria in 2007 to honor Ignatz Rudolph Schiner, the entomologist who first described Wohlfahrtia magnifica [3]. More recent reports suggest that Ignatzschineria is not exclusively associated with Wohlfahrtia species and may be transmitted by other flesh flies as well [2]. Although Ignatzschineria sp. are generally difficult to isolate in routine laboratory procedures, they can be identified by $16 \mathrm{~S}$ rRNA gene amplification and sequencing [1]. Human infections with pathogenic I. indica are uncommon and have only been described in case reports. When pathogenic, I. indica is relatively susceptible and can often be eradicated with a short course of oral antibiotics [2]. Here, we describe a case of I. indica bacteremia in a man with decubitus ulcers infested with maggots.

\section{Case Report}

A 46-year-old African American male $(108.9 \mathrm{~kg})$ with a past medical history of hypertension, stage 2 chronic kidney disease, and gout was brought to the emergency department by paramedics after being found unresponsive and hypotensive at home. The spouse of the patient called paramedics because the patient's breaths were shallow, and he was unresponsive. Chest compressions were initiated because paramedics could not feel a pulse. The patient was then intubated, and intravenous fluids and dopamine were given for hypotension.

On arrival to the emergency department, the patient was found to be hypotensive and hypothermic with a blood 


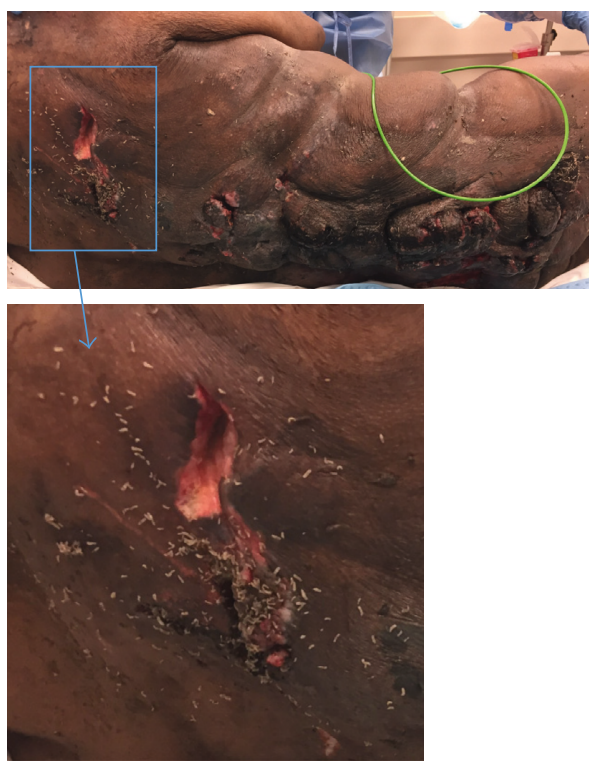

FIGURE 1: Our patient presented with neglected back wounds infested with maggots that ultimately may have been the source of Ignatzschineria indica in the bloodstream.

pressure of $63 / 36 \mathrm{mmHg}$ and a temperature of $30.9^{\circ} \mathrm{C}\left(87.6^{\circ} \mathrm{F}\right)$. Other vital signs included heart rate of 70 beats per minute, respiratory rate of 16 breaths per minute (intubated), and oxygen saturation of 99\%. Physical exam was significant for multiple laceration-type lesions that were infested with maggots along the back covering the thoracic, lumbar, and sacral levels (Figure 1). A stage 4 sacral decubitus ulcer and an ulceration of the left heel were also noted on exam. Reportedly, the patient was bedbound on his couch for 6-8 months due to significant pain attributed to gout along with depression.

The patient was found to have lactic acidosis, hyperkalemia, leukocytosis, significant anemia, and acute kidney injury (Table 1). Urinalysis was positive for leukocyte esterase and had $>100$ white blood cells per high power field (40x objective). Blood and urine cultures were obtained, and the patient was given intravenous fluid, norepinephrine, intermittent vancomycin $1750 \mathrm{mg} \mathrm{IV}$, and piperacillintazobactam $3.375 \mathrm{~g}$ IV every 6 hours. The patient's hyperkalemia was treated medically; a blood transfusion was ordered for his anemia. The patient was stabilized with an improving blood pressure and was admitted to the medical intensive care unit for shock, attributed mostly to sepsis.

Initial blood cultures grew Streptococcus gallolyticus, Streptococcus anginosus, and an undetermined Gramnegative rod species. Urine cultures grew oxacillinsusceptible Staphylococcus aureus. Wound cultures from back grew Proteus mirabilis and diphtheroids. The undetermined Gram-negative was sent to Mayo Clinic Laboratories for further identification and was eventually identified as Ignatzschineria indica, susceptible to amikacin, aztreonam, cefepime, ceftazidime, ciprofloxacin, gentamicin, levofloxacin, meropenem, piperacillin-tazobactam, and tobramycin. On day 7, piperacillin-tazobactam was changed
TABle 1: Pertinent laboratory results.

\begin{tabular}{lc}
\hline Lab & Result \\
\hline Bicarbonate (point of care) & $8 \mathrm{mmol} / \mathrm{L}$ \\
Hemoglobin & $4 \mathrm{~g} / \mathrm{dL}$ \\
Lactate (point of care) & $>16 \mathrm{mmol} / \mathrm{L}$ \\
$\mathrm{pH}$ (arterial blood gas) & 7.01 \\
Potassium (point of care) & $6.9 \mathrm{mmol} / \mathrm{L}$ \\
Serum creatinine & $3.1 \mathrm{mg} / \mathrm{dL}$ \\
White blood count & $64,300 / \mathrm{mL}(84 \%$ neutrophils $)$ \\
\hline
\end{tabular}

to cefepime $2 \mathrm{~g}$ IV every 12 hours and metronidazole $500 \mathrm{mg}$ IV every 8 hours due to an increase in white blood cell count and worsening renal function.

A surgery team performed debridement of the back wounds and obtained cultures that grew Escherichia coli and Proteus mirabilis. A tissue sample from the sacrum was sent to pathology and was found to show acute osteomyelitis. Following surgery and antimicrobial therapy, the patient's clinical status improved. After susceptibilities for I. indica returned, vancomycin was discontinued on day 8 , narrowing antimicrobial treatment to cefepime and metronidazole. On day 10, metronidazole was discontinued. After approximately 17 days of cefepime, treatment was changed to renally dosed oral levofloxacin (750 mg every 48 hours). The Infectious Diseases team recommended this treatment to be continued for 6 weeks from the initiation of cefepime in order to complete a course for osteomyelitis.

\section{Literature Review}

We searched PubMed with the keyword Ignatzschineria (17 results), Ignatzschineria indica (2 results), and Schineria (13 results) in June 2017 and found 7 case reports. In the first report in 2007, a homeless male presented with mild fever, increased C-reactive protein, normal white blood count, and wounds invaded by maggots [4]. His wound samples grew Proteus mirabilis, group A and group G streptococci, Morganella sp., Bacteroides fragilis, and Candida albicans. His blood culture was positive for a Gram-negative rod strain ADV4155.05, which was later identified as Schineria species. The strain was susceptible to beta-lactams, aminoglycosides, fluoroquinolones, erythromycin, rifampin, and colistin. Local debridement, bandaging, use of sulfadiazine, and ofloxacin $400 \mathrm{mg}$ /day PO plus cefotaxime $6 \mathrm{~g} /$ day IV for 2 weeks resulted in clinical improvement. The patient was discharged with ciprofloxacin $500 \mathrm{mg} /$ day PO plus amoxicillin-clavulanic acid $3 \mathrm{~g}$ /day PO for 20 days.

In 2014, three cases of Ignatzschineria indica-associated myiasis were published [2]. In the first of these three reports, a homeless male presented with a painful left foot that had been injured in a motor vehicle accident 2 months prior. He had been unable to treat his wounds or change the dressings since the accident. Upon undressing the wound, maggots were visible in the wound and between the digits. He had elevated C-reactive protein and a normal white blood cell count. Empiric therapy was started with ampicillinsulbactam $3 \mathrm{~g}$ IV every 6 hours and vancomycin $1.25 \mathrm{~g} \mathrm{IV}$ 
TABle 2: Reported cases of Ignatzschineria indica as a human pathogen.

\begin{tabular}{lccccccc}
\hline Reference & $\begin{array}{c}\text { Year } \\
\text { published }\end{array}$ & $\begin{array}{c}\text { Patient } \\
\text { sex }\end{array}$ & $\begin{array}{c}\text { Patient } \\
\text { age (yrs) }\end{array}$ & $\begin{array}{c}\text { Geographical } \\
\text { region/location }\end{array}$ & $\begin{array}{c}\text { Culture } \\
\text { source }\end{array}$ & Treatment & Outcome \\
\hline Roudiere et al. [4] & 2007 & M & 39 & $\begin{array}{c}\text { Montpellier, } \\
\text { France }\end{array}$ & Blood & $\begin{array}{c}\text { Ofloxacin plus cefotaxime then } \\
\text { ciprofloxacin plus amoxicillin- } \\
\text { clavulanate }\end{array}$ & $\begin{array}{c}\text { Discharged to addiction } \\
\text { treatment center }\end{array}$ \\
\hline Barker et al. [2] & 2014 & M & 64 & $\begin{array}{c}\text { Kentucky, } \\
\text { United States }\end{array}$ & Blood & $\begin{array}{c}\text { Ampicillin-sulbactam plus } \\
\text { vancomycin then cephalexin }\end{array}$ & $\begin{array}{c}\text { Discharged, lost to } \\
\text { follow-up }\end{array}$ \\
\hline Barker et al. [2] & 2014 & M & 67 & $\begin{array}{c}\text { South Dakota, } \\
\text { United States }\end{array}$ & Blood & $\begin{array}{c}\text { Piperacillin-tazobactam plus } \\
\text { clindamycin then ciprofloxacin } \\
\text { and vancomycin }\end{array}$ & Discharged \\
\hline Barker et al. [2] & 2014 & M & 26 & $\begin{array}{c}\text { Texas, United } \\
\text { States }\end{array}$ & Urine & Data unavailable & Data unavailable \\
\hline Brun et al. [5] & 2015 & M & 69 & $\begin{array}{c}\text { Loire Valley, } \\
\text { France }\end{array}$ & Blood & Ceftriaxone & Death \\
\hline Heddema et al. [6] & 2016 & M & 71 & $\begin{array}{c}\text { Sittard-Geleen, } \\
\text { Netherlands }\end{array}$ & Blood & Amoxicillin-clavulanate & $\begin{array}{c}\text { Discharged, good } \\
\text { clinical response }\end{array}$ \\
\hline Mejias et al. [7] & 2016 & F & 76 & $\begin{array}{c}\text { Pennsylvania, } \\
\text { United States }\end{array}$ & $\begin{array}{c}\text { Breast } \\
\text { abscess }\end{array}$ & $\begin{array}{c}\text { Vancomycin plus piperacillin- } \\
\text { tazobactam then piperacillin- } \\
\text { tazobactam }\end{array}$ & Discharged \\
\hline
\end{tabular}

every 12 hours, necrotic tissue was debrided, and the third digit was amputated. Blood cultures were positive for nonhemolytic Gram-negative short plump rods that produced a "yellowish" pigment on blood agar. The organism was identified as Ignatzschineria (Schineria) indica. The patient was started on cephalexin $500 \mathrm{mg}$ PO three times daily, discharged on day 3 , and lost to follow-up.

In the second of three cases, a male with chronic alcoholism and extremely poor hygiene was admitted with chronic nonhealing ulcers in the left heel with maggot infestation and foul-smelling purulent discharge [2]. He was treated empirically with piperacillin-tazobactam IV and clindamycin IV. Blood cultures from the day before admission from an outside hospital grew Streptococcus pyogenes and Ignatzschineria indica. The latter isolate was susceptible to amikacin, gentamicin, tobramycin, cefepime, aztreonam, ciprofloxacin, levofloxacin, ticarcillin-clavulanate, and meropenem. The patient received a below-the-knee amputation and was treated with ciprofloxacin $500 \mathrm{mg}$ PO twice daily and vancomycin $1 \mathrm{~g}$ IV every 12 hours for 2 weeks after the surgery.

In the final of the three cases, a paraplegic male was admitted for complications from a previous gunshot wound, including nonhealing decubitus ulcers and multiple past hospital admissions for urinary tract infections [2]. His urine cultures had been positive for Escherichia coli, Proteus mirabilis, vancomycin-resistant Enterococcus faecalis, Pseudomonas aeruginosa, Providencia stuartii, and on one occasion, an unidentifiable Gram-negative rod, which was later identified as Ignatzschineria indica. It was susceptible to aztreonam, ceftriaxone, cefepime, gentamicin, meropenem, trimethoprim-sulfamethoxazole, and tobramycin. It was unclear whether the patient received any treatment.

In 2015, a case reported the association of necrotizing wounds colonized by maggots with Ignatzschineria-associated septicemia in a man found unconscious in a forest [5]. He presented with cardiorespiratory arrest, cyanosis of the extremities, a necrotic skin lesion on the right shoulder, and many maggots around the genital organs. The patient was treated empirically with ceftriaxone IV. Blood cultures revealed Enterococcus faecalis, Enterobacter cloacae, Providencia stuartii, Corynebacterium spp., and a Gramnegative bacillus that was later identified as Ignatzschineria ureiclastica. The I. ureiclastica isolate was susceptible to all beta-lactams, aminoglycosides, fluoroquinolones, colistin, and trimethoprim/sulfamethoxazole. Ten days after hospital admission, the patient was found dead in his bed from no apparent cause, despite recent clinical improvement.

In a similar case reported in 2016, a man with a history of alcohol and nicotine abuse and COPD was admitted after being found unconscious and hypoxemic in front of his house. Results showed an elevated white blood cell count, C-reactive protein, and serum glucose [6]. Physical examination revealed a wound between his first and second toe on his right foot in which a great number of maggots were present. He was initially treated with steroids, bronchodilators, and amoxicillin/clavulanic acid. Blood cultures drawn upon admission revealed a Gram-negative, oxidase-positive, aerobic, catalase-positive rod later identified as Ignatzschineria (species not known) that was susceptible to amoxicillin/ clavulanic acid and ciprofloxacin. It was found to be beta-lactamase positive. The wound was treated with povidone-iodine and melolin dressings, and a two-week course of amoxicillin/clavulanic acid was completed with good clinical response.

Finally, a female with a history of hypertension and diabetes presented with intense abdominal pain, constipation, and an elevated white blood cell count [7]. Physical examination revealed a left side fungating breast mass, malodorous necrotic tissue on the left nipple, and a left axillary abscess. She was empirically started on vancomycin IV and piperacillin/tazobactam IV. Cultures from abscess fluid drawn five days after admission revealed Gramnegative rods identified as Proteus penneri, Providencia 
stuartii, and Ignatzschineria indica (susceptible to amikacin, aztreonam, ceftazidime, ciprofloxacin, imipenem, levofloxacin, meropenem, trimethoprim/sulfamethoxazole, and tobramycin). The patient was treated for 14 days with piperacillin/tazobactam, resulting in reduced leukocytosis. She was discharged and referred to hematology/oncology.

\section{Discussion}

Over the past 10 years, there have been a handful of cases reported where Ignatzschineria indica was considered to be pathogenic [2, 4-7]. There are several similarities between case reports, predominantly wound infestation with maggots. This is not unexpected, as Ignatzschineria may be transmitted by multiple fly species, including the parasitic flesh fly, as described above $[1,2]$.

Similar to the cases presented by Roudiere, Barker, Brun, and Heddema, our patient was a male with poor hygiene who presented with a neglected wound infested with maggots, fever, elevated white blood count, and elevated C-reactive protein. As in five of the seven reported cases, our patient had polymicrobial culture results. In five cases, I. indica that was fairly susceptible to antibiotics was grown from blood cultures, similar to our patient.

Our patient received a longer course of antibiotic coverage compared to the other reported cases due to the evidence of bone involvement on pathology. Each patient presented with a varying degree of severity, resulting in differing outcomes. Table 2 gives a summary of the cases, along with the prescribed treatments.

This compilation of cases demonstrates the need for clinicians to be aware of possible Ignatzschineria infection in patients presenting with poor hygiene and presence of maggots. Medical staff should consider collecting samples of maggots from these infested wounds for further evaluation by professional entomologists in order to better understand the transmission of $I$. indica.

\section{Conflicts of Interest}

The authors declare that there are no conflicts of interest regarding the publication of this paper.

\section{References}

[1] A. K. Gupta, M. S. Dharne, A. Y. Rangrez et al., "Ignatzschineria indica sp. nov. and Ignatzschineria ureiclastica sp. nov., isolated from adult flesh flies (Diptera: Sarcophagidae)," International Journal of Systematic and Evolutionary Microbiology, vol. 61, no. 6, pp. 1360-1369, 2011.

[2] H. S. Barker, J. W. Snyder, A. B. Hicks et al., "First case reports of Ignatzschineria (Schineria) indica associated with myiasis," Journal of Clinical Microbiology, vol. 52, no. 12, pp. 4432-4434, 2014.

[3] E. M. Tóth, A. K. Borsodi, J. P. Euzéby, B. J. Tindall, and K. Márialigeti, "Proposal to replace the illegitimate genus name Schineria Tóth et al. 2001 with the genus name Ignatzschineria gen. nov. and to replace the illegitimate combination Schineria larvae Tóth et al. 2001 with Ignatzschineria larvae comb. nov," International Journal of Systematic and Evolutionary Microbiology, vol. 57, no. 1, pp. 179-180, 2007.
[4] L. Roudiere, H. Jean-Pierre, C. Comete, I. Zorgniotti, H. Marchandin, and E. Jumas-Bilak, "Isolation of Schineria sp. from a man,” Emerging Infectious Diseases, vol. 13, no. 4, pp. 659-661, 2007.

[5] C. L. Brun, M. Gombert, S. Robert, E. Mercier, and P. Lanotte, "Association of necrotizing wounds colonized by maggots with Ignatzschineria-associated septicemia," Emerging Infectious Diseases, vol. 21, no. 10, pp. 1881-1883, 2015.

[6] E. Heddema, F. Janssen, and H. van Westreenen, "A case of Ignatzschineria bacteraemia in an unconscious man from the Netherlands," JMM Case Reports, vol. 3, no. 3, p. e005043, 2016.

[7] L. Mejias, C. Curcio, A. Sanchez et al., "Ignatzschineria indica isolated from a human breast abscess: a rare case," Journal of Medical Cases, vol. 7, no. 11, pp. 502-505, 2016. 


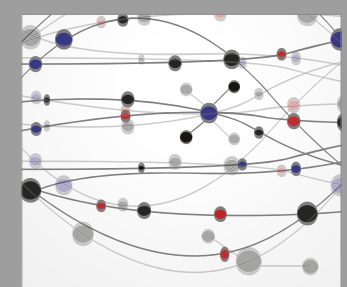

The Scientific World Journal
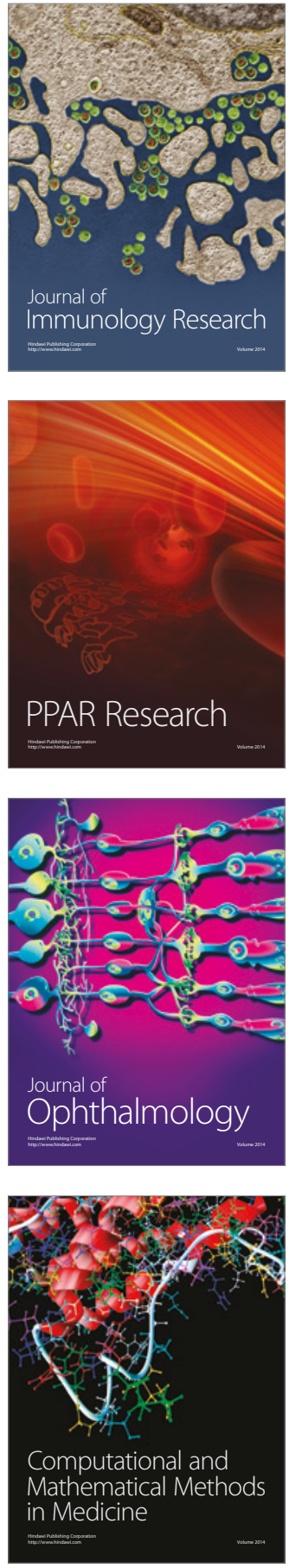

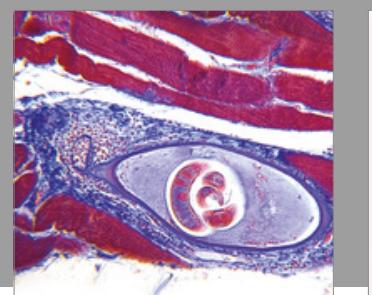

Gastroenterology Research and Practice
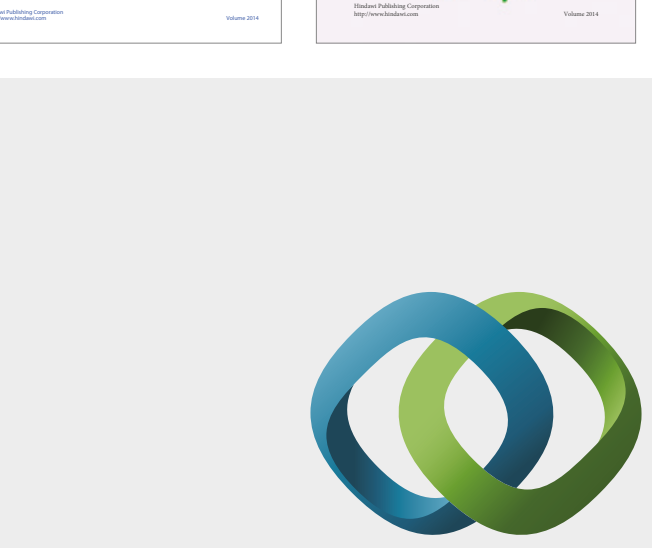

\section{Hindawi}

Submit your manuscripts at

https://www.hindawi.com
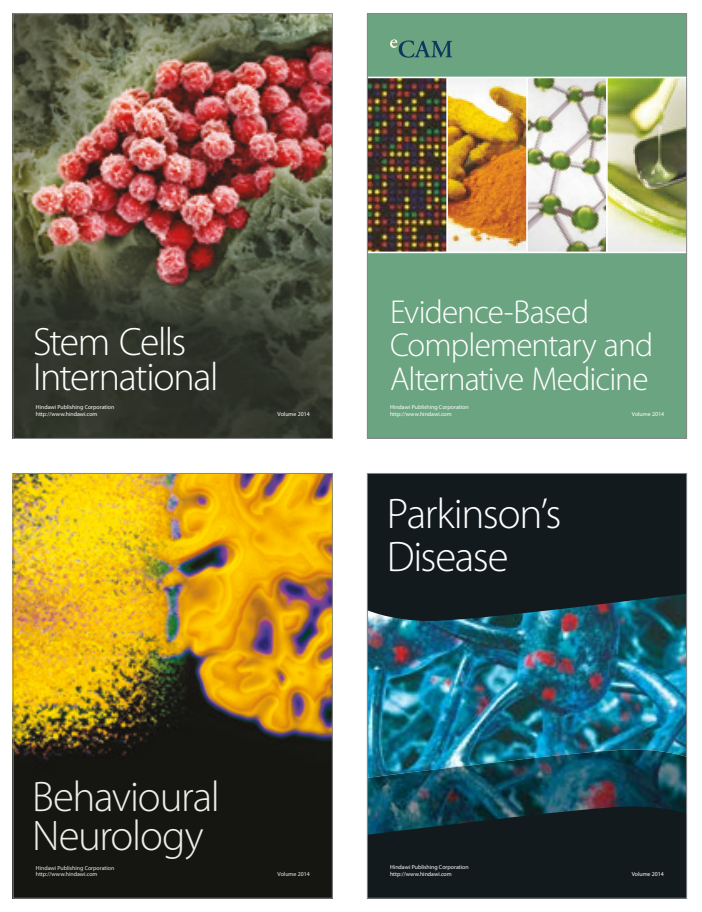
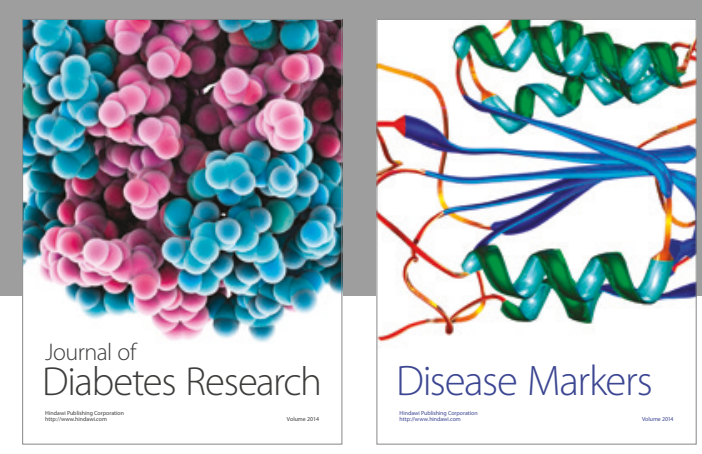

Disease Markers
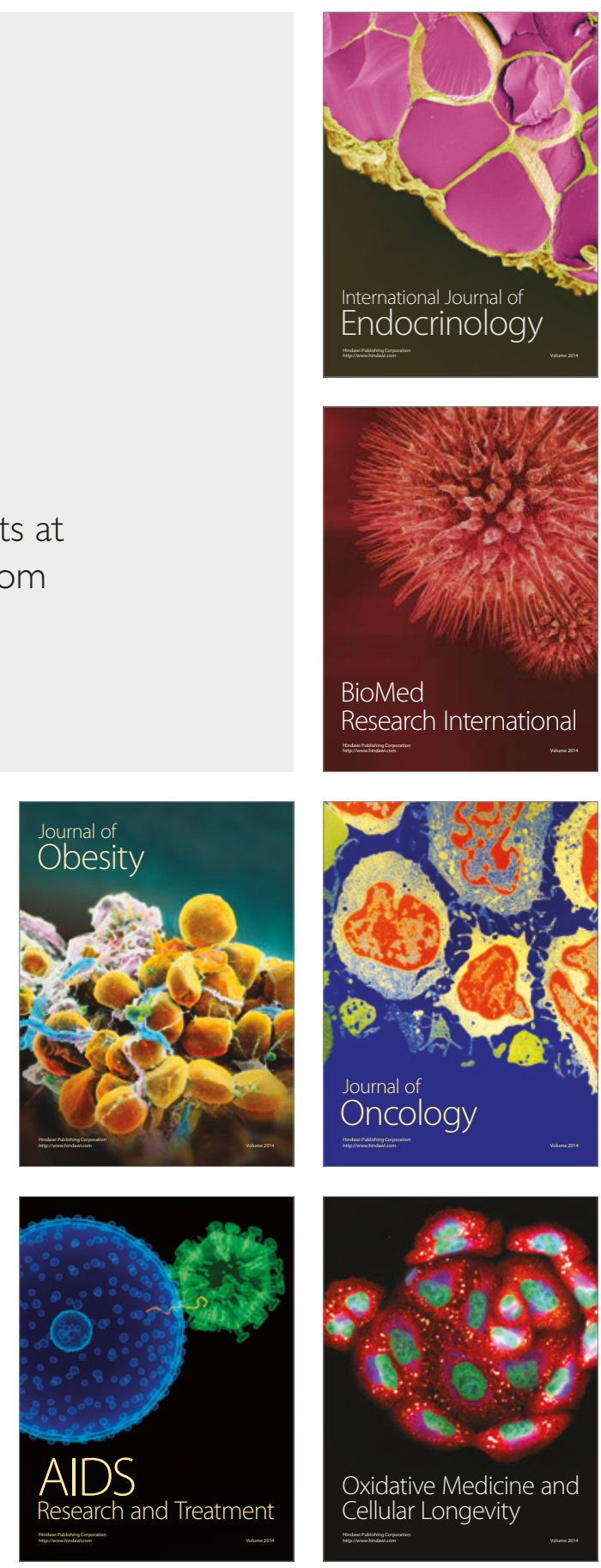